\title{
Addressing the Emotional Distress of Healthcare Workers: Creating a Cohesive Resiliency Program Response to COVID-19
}

\author{
K Gilrain*, P Fizur, R Allen, E Campbell, P Watson, S Jordan, E Kupersmith, A Rostain \\ Division of Behavioral Medicine, Cooper University Hospital, USA
}

\section{Article Info}

\section{Article Notes}

Received: July 15, 2020

Accepted: August 11, 2020

\section{*Correspondence:}

Dr. Kelly L. Gilrain, Ph.D., Division of Behavioral Medicine,

Cooper University Hospital, One Cooper Plaza, D222, Camden, New Jersey 08103, USA; Telephone No: 856-342-3129; Email: Gilrain-kelly@cooperhealth.edu.

(c) 2020 Gilrain K. This article is distributed under the terms of the Creative Commons Attribution 4.0 International License.

\section{Keywords:}

Resiliency

Wellness

Burnout

COVID-19

\section{Abstract}

Background: Promoting resilience in healthcare workers is a well-studied area that has taken on new significance in the context of the COVID-19 pandemic. Extant literature suggests a need to expand resiliency efforts, with a recommended focus on multimodal approaches.

Purpose: The present account describes the unique challenges faced by one urban, northeastern academic medical center during the COVID-19 crisis in promoting resiliency in their healthcare workers at all levels, with the related aim of examining feasibility and acceptability of doing so.

Methods: A review of existing procedures targeting wellness and resiliency at this hospital was completed. A literature review was conducted with regard to promoting resiliency and preventing burnout, with emphasis placed on case studies from other institutions during the pandemic. Post-intervention surveys were conducted to assess feasibility and acceptability.

Results: A multi-component approach was created based on a review of the literature and all available information. The current report focuses on the first five weeks of that effort, the results of which suggest sufficient feasibility and high acceptability among those surveyed. Common themes raised in support sessions are also identified and discussed.

Conclusions: The challenges raised by COVID-19 are significant, with a high probability of impact on the wellbeing of health care workers. Targeting resiliency now may be a key factor in preventing pathological responses later. The current approach appears feasible and acceptable with regard to targeting key resiliency areas. Future studies should focus on the longer term outcomes of these efforts.

When traumatic events arise on the national or global level, mental health providers have a specific and important role to play in minimizing the psychological impact on those affected either as directed victims or first responders. As described in Pandya (2013), psychological interventions offered in the wake of the September $11^{\text {th }}$ attacks included acute services such as debriefings and other single-time before shifting to post-acute interventions such as community outreach targeting normative psychological sequelae, standard evidence-based protocol for trauma, and hospital-based psychiatric services. What arose in the weeks, months, and years afterward most closely resembled psychological first aid (PFA) and was shown to be effective. Expanded up by Haugen, Splaun, Evces, and Weiss (2013), among the most effective interventions included an emphasis on meaning making surrounding the traumatic event; focus on the most affect-laden components of the traumatic exposure; and identifying and challenging the implicit strategies used by 
individuals to avoid discussion of components of their traumatic memories and the attendant negative affect. It was with these learned lessons in mind that the presently described interventions are framed.

On Sunday March 22, 2020 members of the departments of Psychiatry and Behavioral Medicine at Cooper University Health Care (CUHC) were called by senior leadership to provide direct support to frontline healthcare workers in the ICU and med/surg units who were finding it difficult to get through their shifts knowing that the first suspected and confirmed cases COVID-19 had been admitted to their floors. Two health psychologists, both trained from a CBT perspective and employed at Cooper on the Behavioral Medicine Service and an AVP visited 4 units and provided brief psychosocial interventions to ease the collective distress of a total of 60 providers and support personnel. Sessions included psychoeducation on anxiety, normalization and validation of individual emotions, and practical skills focused on grounding techniques, mindfulness, and acceptance-based strategies. These efforts were repeated, expanded upon and formalized into a task force designated the Resiliency Resources Team (RRT). The present account describes the unique challenges faced by CUHC and the State of New Jersey during the COVID-19 crisis, summarizes responses by similar groups across the country, describes Cooper's approach to wellness promotion, and explains how this has informed the work of the RRT. The authors offer recommendations for those engaged in similar efforts in other health care institutions.

\section{Cooper University Health Care}

Cooper University Health Care consists of a 635-bed hospital located in Camden, NJ, and is a tertiary care, academic, level 1 trauma center with over 100 ambulatory clinics, the MD Anderson Cancer Center at Cooper, 3 urgent care centers, and affiliated with the Cooper Medical School at Rowan University all of which serve the greater southern New Jersey region. Cooper University Hospital (CUH) is the only level 1 trauma center in Southern New Jersey. In total, Cooper's 7,000 providers, 600 physicians, 300 APP's, 325 Physicians in residency and fellowship and our staff care for nearly 2 million patients per year, many of whom live in disadvantaged areas in Camden.

\section{COVID-19 in New Jersey}

On March 9, 2020, New Jersey declared a state of public health emergency related to the novel coronavirus outbreak. By March 16, mass gathering restrictions and small businesses closures were in effect. On March 17, CUHC instituted a temporary visitor restriction policy. Schools were closed on March $18^{\text {th }}$ and a stay at home order was initiated on March $21^{\text {st }}$. As these events unfolded, CUHC providers quickly began to report increased psychological distress in the patients for whom they were caring as well as each other. This distress rapidly intensified, which raised alarm with their supervisors and the senior executive team. Concerns focused on the immediate and long-term consequences of the virus and the impact of these protective orders across all domains of their lives. On March $26^{\text {th }}$, Cooper was designated by the NJ State Health Commissioner to be one of three coordinating entities to manage surge planning, and the leader for these efforts in Southern New Jersey.

\section{Responses at Other Academic Medical Centers}

To ensure that empirically supported and clinically sound interventions were incorporated into program development, the RRT utilized available literature on provider wellness and employee health. Updated articles and information from COVID-19 hotspots helped to identify what emotional responses could be expected and offered recommendations for improving provider and staff resiliency.

At Mount Sinai Health System in New York, the epicenter of COVID-19 in the United States, the efforts of Berg et al $(2020)^{9}$ focused on basic needs, psychosocial needs and mental health to enhance well-being and reduce additional stress of their providers during the pandemic. Physicians from Stanford University, leaders in the area of Provider Wellness, conducted eight listening sessions with groups of 69 providers during the first week of the COVID-19 pandemic to assess specific concerns ${ }^{1}$. Recommendations fall into categories of "Hear $\mathrm{Me}$, Protect Me, Prepare Me, Support Me and Care for me" and were offered to institutional leadership. The University of Kansas Medical Center (KUMC) created several well-received initiatives to support provider emotional well-being ${ }^{10}$. This medical center, immediately created a request for wellness/ resiliency supports, initiated psychological first aid with professional teams, identified a pool of KUMC psychologists and chaplains to provide telephone supports and in person supports. They also initiated 5-10 one hour group calls daily from $7 \mathrm{am}-10 \mathrm{pm}$ to provide support to those identified as in highest distress. Providers from the University of Michigan Medical School and from Rutgers University advocated using "micropractices" to encourage providers to engage in wellness practices ${ }^{2}$. Micropractices, as the authors define them, are tools that take minimal time to learn and implement. One such practice is engaging in mindfulness exercise during an activity that a provider often engages in (hand washing, logging into EMR) ${ }^{2}$. The common themes of attending to practical needs, providing emotional support, and facilitating communication were identified among all sources. These themes informed the initial efforts of Cooper's RRT. 


\section{Wellness at Cooper Prior to COVID-19}

Prior to the COVID-19 pandemic, there had been several health and wellness committees throughout the CUHC system, including those born out of the Physician Engagementand the GME offices, which focused on physician burnout and overall well-being. "Compassionomics" written by Cooper physicians, Dr. Stephen Trzeciak and Dr. Anthony Mazzarelli ${ }^{3}$, present scientific evidence which supports the fact that human connection and caring makes a significant difference in the lives of others. The authors' note that having compassion for others also has a direct positive impact on one's sense of well-being and can be the antidote for burnout ${ }^{4-8}$. The Behavioral Medicine team had proposed an evidence-based, multi-component wellness program to target a broader segment of hospital staff at the individual, leadership, and organizational levels. Components included active interventions (i.e. grounding and mindfulness skills) and passive cues (i.e. posters reminding teams of learned skills on nursing floors and clinics) to enhance connectivity among providers and staff. The primary aim of the initiative was to examine resiliency, perceived organizational support, compassion and professional fulfillment. This framework, already in place when the pandemic hit, has served as the conceptual foundation for the multi-pronged resiliency program delivered by the RRT.

\section{Cooper Resiliency Resources Team (RRT)}

The core group of the RRT at Cooper is comprised of three psychologists from our Behavioral Medicine team, our Chief of Psychiatry, a Master's Level Clinician from our EAP, our AVP of APP's and ILP's with administration and coordination support from Human Resources and Organizational Development. We recruited other volunteer clinicians from around the health system including psychiatrists, psychologists, LCSW's and LPC's from our Psychiatry, Behavioral Medicine, Addictions and Transitional Navigator teams, who were willing to provide on-site groups across the health system on a rotating schedule (4 hours per week). Most individuals were agreeable to engaging in this endeavor but some clinicians approached declined due to their own anxiety about being onsite with providers working with COVID patients. The primary goal of the programming offered by the RRT is to provide emotional and social support and education through in-person brief resiliency workshops, peer support groups, and digital content.

\section{Methods}

\section{In-person Resiliency Workshops}

The RRT recognized that in-person support group meetings would be vital to the success of the program. Requests were for immediate and in person, on site emotional supports. The need for these specific types of groups is supported by the success found in Balint Groups. Balint Groups were created, and became widely activated, in the 1970's as a collaborative medical group of clinicians and teachers who met to discuss a variety of challenges faced by physicians. Typically these groups are led by a physician and a psychologist to examine the relationship between doctor and patient in ordinary consultations and interactions including the emotional and personal understanding of this relationship by discussing cases. Research supports the efficacy of these groups in reducing burnout and depressive symptoms. Specifically, Popa-Velea et al $(2019)^{12}$ finds that Baling training improved scores on global burnout including emotional exhaustion and depersonalization, alexithymia and perceived social support.

Clinicians in this study were thus identified and those who joined the RRT agreed to a commitment of four hours per week on a rotating six-week schedule. Sessions were led by two RRT team members and are open to all employees. Individuals, Leaders and Groups would submit a request through the Wellness Website with additional information about the team's needs. A brief discussion of the stress response and the typical processing of emotional reactions opens the session. The facilitators would then identify the most salient needs of the group to inform interventions offered in the second half of the session. Apart from these components of normalization and education, RRT facilitators provide education and practice of mindfulness skills, acceptance-based skills, and grounding techniques. Practical suggestions such as reducing media intake and enhancing social connectivity during this time are discussed. A brief post-session survey is sent to participants to guide future program development for the COVID-19 crisis and beyond. Data collected includes number of attendees, themes observed, requests and concerns, resolution, and positive outcomes which is summarized and provided to senior leadership.

\section{Digital Content}

Prior to the pandemic, the website http://wellness. cooperhealth.org/ had been created to act as a central resource for all health and wellness initiatives across the health system. New sections of this site were created for COVID-19 related content. Based on a common, opensource content management system (CMS), the site and its underlying framework allowed for the rapid development of the previously described print, audio, and video resources. This framework also afforded the RRT a central resource for initial data collection to assess the feasibility and acceptability of these efforts. Since the initiation of the RRT, this site has become dedicated to relevant content. More specifically, recognizing that not all providers and staff would be able to attend an in-person resiliency group, and that those who did would likely want additional 
resources following the session, an archive of resources has been uploaded onto the CUH Wellness Website. Additionally, some providers and staff who may be at home during part of this challenging time would have access to these resources as well. More specifically, a resource page was created and divided into those applicable to the general public, to parents and children, and those specific to medical professionals focusing on practical aspects such as adapting to telemedicine, engaging in self-care, and a repository of open-access publications relevant to the pandemic. The media section includes audio and video recordings from members of the RRT discussing coping skills for pandemic-related stressors. A podcast was created, modeled after the in-person session and designed both to help train new facilitators and to help members of the Cooper community who are not able to attend a live session. Finally, the website hosts the Daily Dose, inspirational and informative messages posted at 8am daily.

\section{Peer Support Teams}

A theme that consistently emerged in the early inperson sessions was that, while support from the RRT and the CUH administration was generally appreciated, those on the individual floors and ambulatory offices have unique perspectives on their group's reactions. Peer support groups have been created to connect physicians to physicians, nurses to nurses, and advanced practice providers (APP) to APPs. A training manual and associated video resources were created and distributed reviewing basic principles of providing peer support, as well as what to do in situations where a peer may need a greater level of support.

A feedback survey was created to anonymously document the frequency and utility of these groups, as well as to assist in addressing any risk issues that may arise.

\section{Collaboration Across the Health System}

The numerous procedural and cultural shifts across US health care in response to the pandemic has meant that clear communication and frequent, reliable updates are of utmost importance to healthcare providers (Shanafelt, Ripp \& Trockel, 2020) We are fortunate that CUH senior leadership immediately focused on communication about COVID-19 preparedness. CUH co-CEOs and other senior leaders were transparent about Cooper's plans and changes in workflow as they occurred. A daily CEO update email was launched and our Chief Physician Executive created a nightly "Five at Five" WebEx briefing to provide updates regarding hospital admissions, virus testing, IT expansions with telehealth, surge plans, and community connections. Providers and staff receive special recognition for their dedication and hard work in all areas of the healthcare system. This underscores the important and unique role of leadership in providing support to all members of the organization.

Administrative support was provided by Human Resources to coordinate and track in-person support groups. Employee Assistance Program (EAP) webinars and telehealth sessions were available to all CUHC employees to provide individual and group supports as needed. Employee Health staff tasked with triaging calls from employees exhibiting symptoms of COVID-19 infection and quarantined at home received training in the use of the PHQ-2 and 9; employees who self-identified as distressed were referred to the RRT and to EAP. The RRT worked with Marketing and Internal Communications by writing articles for both the broader Cooper community as well as our own teams. The Internal Communication team was invaluable in providing employees with emotional well-being information via daily and weekly updates. The RRT provided assistance to Patient Relations and hospital leadership to address visitor restriction challenges and their effects on our patients' and employees' psychological well-being.

\section{Results}

In the first 30 days, the RRT was able to reach over 600 providers and staff for in person or WebEx support groups. There are additional 500 individuals scheduled for group sessions in the coming month. Units targeted for weekly meetings include the ED, ICU and all COVID-19 positive dedicated units. The RRT has reached out to all members of the CUH community, including teams from Food \&Nutrition, Environmental Services, Facilities, Transport, med surg floors, OBGYN teams, oncology, palliative care, OR and PACU teams, Radiology, Anesthesia, Nursing Leadership and our Incident Management Team to provide resiliency groups.

We began support groups in the hospital and are now reaching out to ambulatory offices. Data from the first wave of support group sessions is summarized in Table 1. In addition, the RRT developed a post resiliency session survey to best understand how in person sessions are impacting our providers and staff, the results of which are summarized in Table 2 . The RRT sought feedback after each session from participants and also asked the group facilitators to summarize themes that arose. As represented in Figure 1, the most common themes that arose included family, work/life balance, bringing the virus home, having sufficient PPE, concerns about frequent change, and overall anxiety. Early responses suggest high acceptability and clear benefit from these efforts. Areas where respondents suggested additional resources be directed include: having dedicated physical space within the hospital to engage in 
Table 1. Cooper Health Care RRT Initial Data

\begin{tabular}{|c|c|c|}
\hline Common Session Themes & Skills Provided & Early Positive Outcomes \\
\hline $\begin{array}{l}\text { Work } \\
\text { - } \quad \text { Heightened anxiety across all areas in hospital } \\
\text { - } \text { Groups reassured having Senior Leadership visible on } \\
\text { - } \quad \text { Concerns about having appropriate PPE } \\
\text { Self Care } \\
\text { - } \quad \text { Ongoing changes in routines } \\
\text { - } \quad \text { Balancing needs of work with needs of family } \\
\text { - No time to decompress between home/work de- } \\
\text { - } \quad \text { Stress Eating } \\
\text { - } \quad \text { Lack of Exercise } \\
\text { - } \quad \text { Maintain a sense of normalcy } \\
\text { Family } \\
\text { - } \quad \text { Cooncern with family members not social distancing } \\
\text { - } \quad \text { Providing enough support to patients who no longer } \\
\text { have visitors } \\
\text { - Concerns related to bringing covid virus home to } \\
\quad \text { families }\end{array}$ & $\begin{array}{ll}\text { - } & \text { Grounding techniques } \\
\text { - } & \text { Mindfulness } \\
\text { - } & \text { Acceptitude } \\
& \text { COVID) } \\
\text { - } & \text { Values Work related to Healthcare } \\
\text { professionals } \\
\text { - } & \text { Meaning Making } \\
\text { - Sleep Hygiene } \\
\quad \text { Social Connections }\end{array}$ & $\begin{array}{l}\text { - Staff recognizing the value of } \\
\text { PPE } \\
\text { Creation of a unit notebook for } \\
\text { all to collaboratively identify } \\
\text { positive reframes } \\
\text { - Social connectedness of the } \\
\text { team and appreciating the op- } \\
\text { portunity to continue interaction } \\
\text { Recognizing food/toiletries were } \\
\text { taken for granted } \\
\text { People are good and are helpful } \\
\text { through the pandemic } \\
\text { Gratitude - Animals are being ad- } \\
\text { opted, having access to food and } \\
\text { basic needs, appreciative of the } \\
\text { food donations additional time } \\
\text { at home spent with immediate } \\
\text { family, for the support from their } \\
\text { colleagues, for the support from } \\
\text { senior leadership }\end{array}$ \\
\hline
\end{tabular}

Table 2. Cooper RRT Post Resiliency Session Survey Results

$$
\begin{gathered}
\text { RRT Survey Results } \\
n=74
\end{gathered}
$$

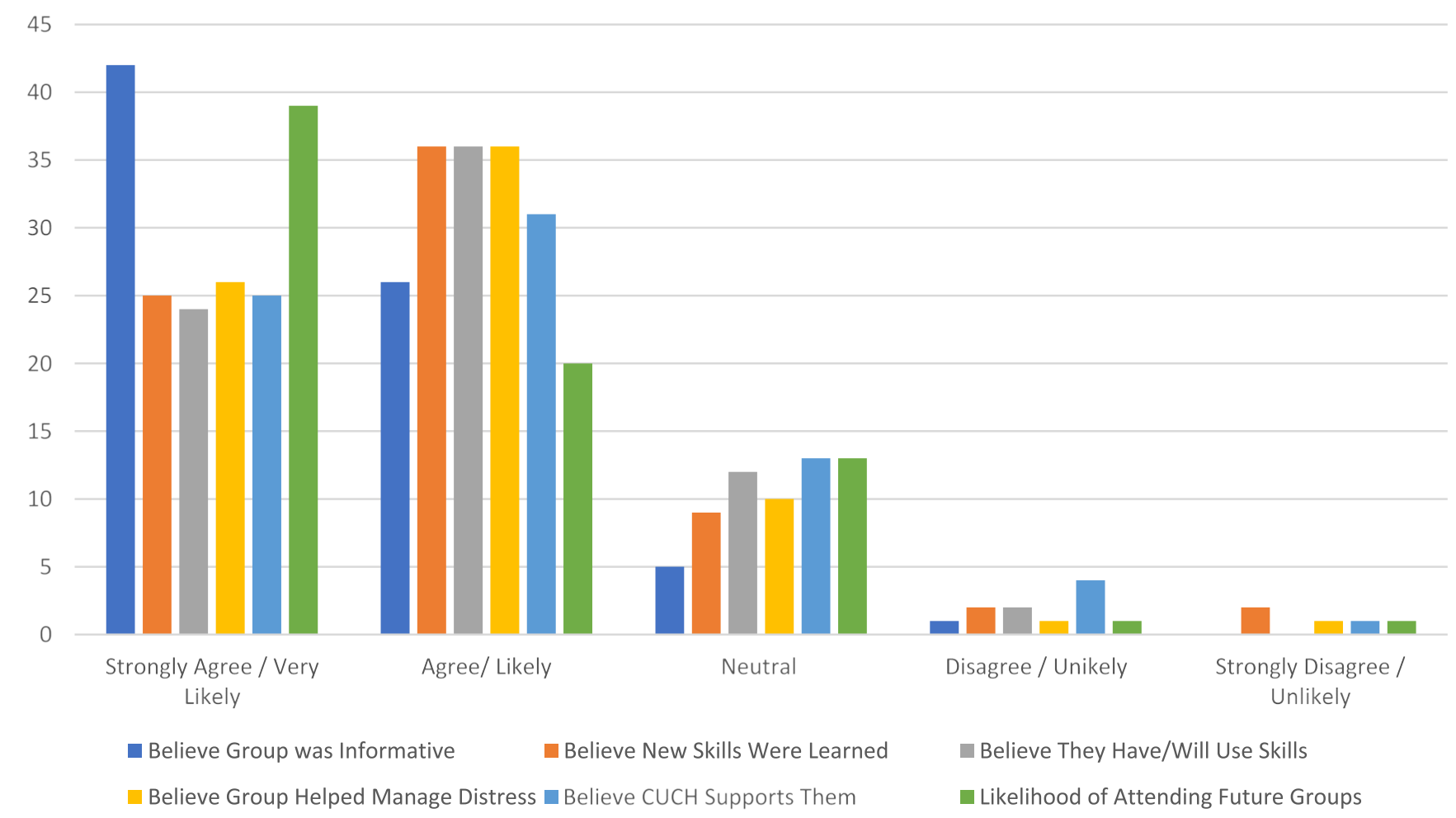

self-care such as mindfulness, providing more guidance to assist employees help their own families when they return home, addressing financial concerns, returning to work after having recovered from COVID-19, helping patients who will be discharged without loved ones at home to support them, acquiring PPE for all staff (e.g. security, transportation, food service), and increasing the length of RRT sessions 


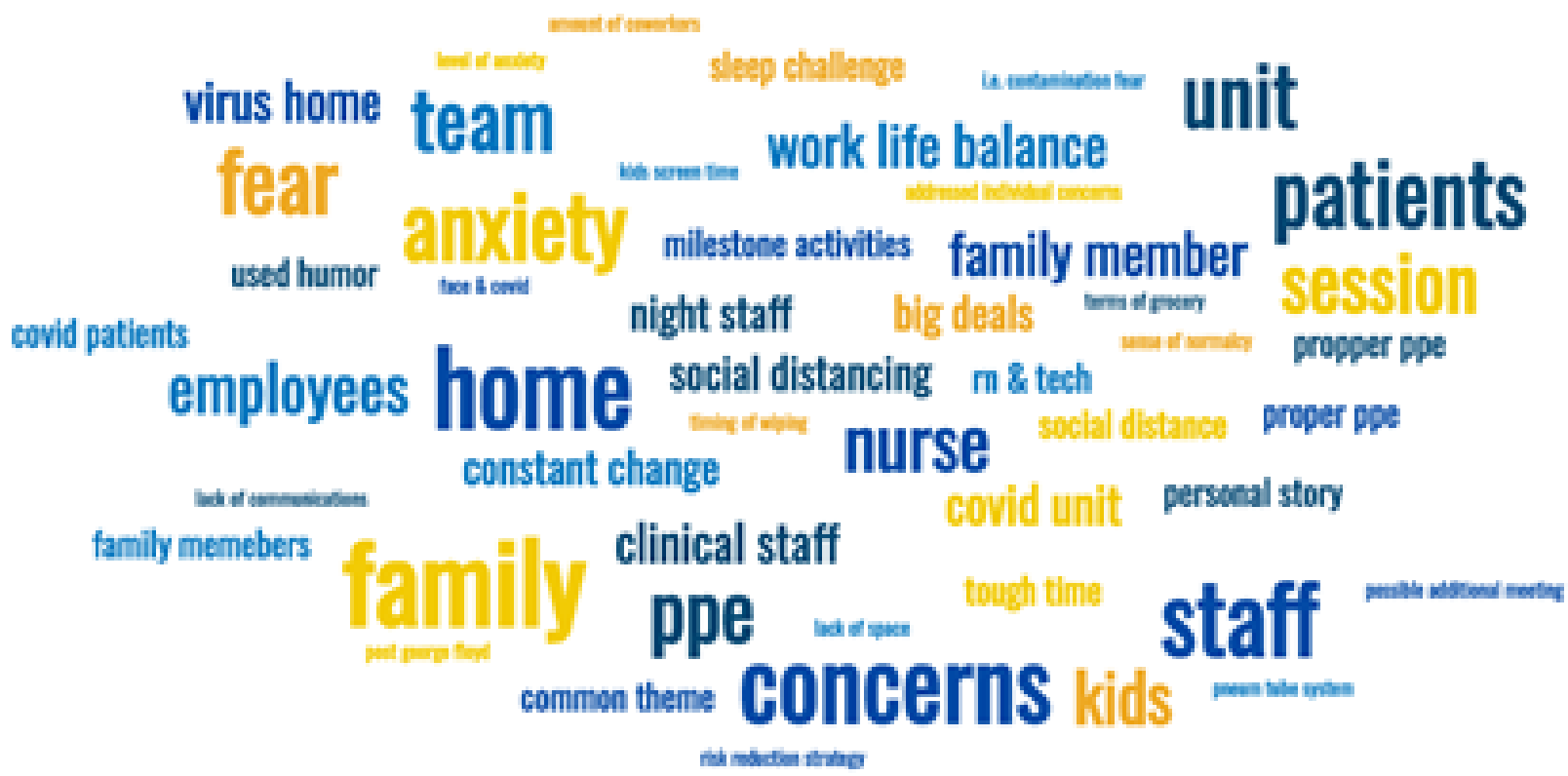

Figure 1. Cooper Health Care RRT Group Themes

The RRT sought feedback after each session from participants and also asked the group facilitators to summarize themes that arose. As represented in Figure 1, the most common themes that arose included family, work/life balance, bringing the virus home, having sufficient PPE, concerns about frequent change, and overall anxiety.

\section{Conclusion}

\section{RRT Through the Pandemic and Beyond}

The overall goals of the RRT are to address anxiety and secondary trauma, ensure resiliency, and promote compassion and post-traumatic growth throughout the COVID-19 pandemic and beyond. While stress can often be overwhelming and provoke psychological distress, it also catalyzes individual transformation and personal growth. RRT clinicians encourage each workshop participant to reflect upon relationships, self-esteem and gratitude as we turn towards compassion and locate a deeper inner wisdom. Research bears out, and our experiences confirm, that discovering a greater sense of meaning can be at the heart of a renewed sense of well-being (Johnston, 2012; Horstman, Kranstuber, Maliski, Hays, Cox, \& Enderle, 2016). Assisting employees working in a health care setting find greater understanding of their life experiences, thinking through new possibilities and identifying personal strengths are all essential to managing the distress brought on by the pandemic. Identifying values, supporting selfexploration and engaging in meaning-making can facilitate positive growth and change during challenging and chaotic times. The RRT will continue to address themes identified in the previous months and in post-session surveys. Focus will be placed on psychoeducation and skills training in the areas of grounding, mindfulness, meaning-making, post-traumatic growth and acceptance. Of note, during the height of the pandemic, focus was on the immediate anxiety and trauma symptoms reported by our colleagues. However, over time, as COVID numbers decreased and care of these patients became more common, other stressors surfaced. Symptoms of overall burnout, challenges in team relationships and interpersonal communication as well as avoidance of emotions or heightened emotionality, somatic complaints and call outs from work were more prevalent. As such, the RRT has had to create a more thorough screening and assessment to best understand the challenges faced by each team prior to engaging in a group. Moreover, prior to an RRT group 'homework' is provided in some cases to set the tone of the group, have those involved be more focused on the goals of the group and to focus on coping skills and styles has been utilized.

As Rosenberg $(2020)^{11}$ points out, resiliency requires deliberate action aimed at developing coping resources on three levels: individual, community and existential. "Communities and individuals facing adversities as diverse as war, famine, poverty, illness, or death do this by harnessing consistent categories of resilience resources." The CUH RRT appreciates the importance of this notion of deliberate resiliency which requires the cultivation of attitudes, skills and relationships to strengthen our individual and collective adaptation to change. Optimistically, there is a constructive "new" normal we are all moving towards. This will include a deeper examination of personal well-being supported by values exploration, seeking opportunities for growth and creating more profound meaning in our day to day lives. 


\section{References}

1. Shanafelt, Tait, Ripp, et al. Understanding and addressing sources of anxiety among health care professionals during the COVID-19 pandemic. JAMA. 2020. Published online April 07, 2020.

2. Fessell, David, Cherniss, et al. (2020). Coronavirus disease 2019 (COVID-19) and beyond: Micropractices for burnout prevention and emotional wellness. Journal of the American College of Radiology. 2020. Published online March 24, 2020.

3. Trzciak S, Mazzarelli A. Compassionomics: The Revolutionary Scientific Evidence that Caring Makes a Difference. 2020.

4. Beaumont E, Durkin M, Hollins Martin CJ, et al. Compassion for others, self-compassion, quality of life and mental well-being measures and their association with compassion fatigue and burnout in student midwives: A quantitative survey. Midwifery. 2016; 34: 239-244.

5. Burnett HJ. Revisiting the compassion fatigue, burnout, compassion satisfaction, and resilience connection among CISM responders. SAGE Open. 2017; 7(3).

6. Cleary M, Kornhaber R, Thapa DK, et al. The effectiveness of interventions to improve resilience among health professionals: $\mathrm{A}$ systematic review. Nurse Education Today. 2018; 71: 247-263.
7. Gonzalez TC, Burnett HJ, Jr, Helm H, et al. An examination of resilience, compassion fatigue, burnout, and compassion satisfaction between men and women among trauma responders (report). North American Journal of Psychology. 2019; 21(1): 1.

8. Kim SR, Park OL, Kim HY, et al. Factors influencing well-being in clinical nurses: A path analysis using a multi-mediation model. Journal of Clinical Nursing. 2019; 28(23-24): 4549-4559.

9. Berg, Sara (2020, April 6). Covid019 front line: Mount Sinai keeps physician well-being in focus. https://www.ama-assn.org/practicemanagement/physician-health/covid-19-front-line-mount-sinaikeeps-physician-well-being.

10. Kurylo, Monica, Kansas University Medical Center. Personal communication, March 27, 2020.

11. Rosenberg AR. "Cultivating Deliberate Resilience During the Coronavirus Disease 2019 Pandemic." JAMA Pediatrics. 2020. April 13, 2020. doi:10.1001/jamapediatrics.2020.1436

12. Popa-Velea O, Trutescu C, Diaconescu L. The impact of Balint work on alexithymia, perceived stress, perceived social support and burnout among physicians working in palliative care: a longitudinal study. Int J Occup Med Environ Health. 2019; 32(1): 53-63. 